\title{
Application of Proteomics in the Discovery of Radiosensitive Cancer Biomarkers
}

\author{
Hui Luo and Hong Ge* \\ Department of Radiation Oncology, The Affiliated Cancer Hospital of Zhengzhou University, Zhengzhou, China
}

Radiation therapy remains an important component of cancer treatment. Gene-encoded proteins were the actual executors of cellular functions. Proteomic was a novel technology that can systematically analysis protein composition and measure their levels of change, this was a high throughput method, and were the import tools in the post genomic era. In recent years, rapid progress of proteomic have been made in the study of cancer mechanism, diagnosis, and treatment. This article elaborates current advances and future directions of proteomics in the discovery of radiosensitive cancer biomarkers.

Keywords: tumor, radiosensitive biomarker, proteomic, application, review

\section{OPEN ACCESS}

Edited by:

Yinsheng Wang,

University of California, Riverside,

United States

Reviewed by:

Weili Miao,

University of California, Riverside,

United States

*Correspondence:

Hong Ge

zlyygehong0199@zzu.edu.cn

Specialty section:

This article was submitted to

Radiation Oncology,

a section of the journal

Frontiers in Oncology

Received: 11 January 2022 Accepted: 04 February 2022

Published: 23 February 2022

Citation:

Luo H and Ge H (2022) Application of Proteomics in the Discovery of Radiosensitive Cancer Biomarkers.

Front. Oncol. 12:852791. doi: 10.3389/fonc.2022.852791

\section{INTRODUCTION}

Radiation therapy is a highly targeted treatment accurately suppressing the tumor with wide ranging application, and contributed to approximately $40 \%$ of all cancer cures across the world (1). This treatment modality is also very effective for other cancer related problems including pain, tumor bleeding, and acute superior vena cava syndrome (2-4). Despite the great benefit, the existence of radiation resistance leads to local recurrence, distant metastasis, and poor survival, this is really a key challenge in cancer treatment (5). With the development of precision medicine in clinic practice, there was an urgent need to screen a number of biomarkers for patient's stratification and thus develop appropriate treatment strategies (6). Indeed, investigators were dedicated to identify potential radiosensitivity biomarkers to perform tailored radiation therapy for cancer patients $(7,8)$. Big data was the basis for the realization of precision medicine (9). After the completion of human genome sequencing, the "post-genome" era has arrived (10). Genes cannot perform the functions by themselves, and their functions need to be performed directly through transcription and translation to proteins (11).

Protein expression can be influenced by environmental conditions, nutritional status, and therapeutic modalities. Protein expression can directly reflect different physiological or pathological processes in the organism (12). Proteomic was an advanced systemic biology research method in the "post-genomic" era, it was studied by large-scale screening and identification of protein expression in body fluids, tissues, cells or organisms under various conditions, and protein-protein interactions will be well recognized (13). Mass spectrometry (MS) has become an important tool for large-scale protein identification in proteomics (14). Currently, research of proteomics mainly focused on basic medicine, health screening, disease prevention, prognosis prediction, patient stratification, and the identification of new targets (15-18).

Since proteins can directly reflect cellular biological processes and were dynamic changed in real time, the application of proteomics in precision medicine has attracted much attention $(19,20)$. 
In recent years, researchers attempt to analyze protein expression in tumor tissue and plasma during radiation therapy by proteomics technology, this will be facilitating the detection of proteins that play a key role during the course of radiotherapy and biomarkers that can predict radiotherapy sensitivity in the earlier phase $(21,22)$. With the development of proteomics, tumor radiation therapy has entered a new era in the context of precision medicine (23). In the present analysis, we reviewed the research progress of proteomics in screening tumor radiotherapy sensitivity markers (Figure 1).

\section{APPLICATION OF PROTEOMICS TO IDENTIFY PREDICTIVE BIOMARKERS OF RADIOSENSIVITY}

Technological advances in MS have driven an increased capability for rapid, accurate and effective proteomic discovery. To date, several proteomics techniques have been applied to identify potential biomarkers of radiosensitivity (Figure 2).

\subsection{Matrix-Assisted Laser Desorption/ Ionization Time-of-Flight Mass Spectrometry (MALDI-TOF-MS)}

MALDI-TOF-MS is an approach in proteomics to identify proteins from simple mixtures frequently combined with two- dimensional gel electrophoresis (24). Relatively less intense sample preparation was required for this technique; besides, peaks in a spectrum were typically used as indications of peptide content. However, reproducibility of outcomes may be a problem because of this technique is sensitivity to contaminants from salts. MALDI-TOF-MS may be replaced with the emerging of advanced MS techniques.

\subsection{Isobaric Tags for Relative and Absolute Quantification (iTRAQ)}

iTRAQ technology is a shotgun-based quantization method that uses isobaric reagents to label all primary amines of peptides and proteins (25). The concentration of potential radiosensitivity biomarkers in cancers can easily be quantified using iTRAQbased proteomics because of the advantages including less time consuming and decreased variation (26). Notably, this technique is expensive, sensitive to contamination from salts, and the variability will arise if the enzymatic digestion is inefficient.

\subsection{Liquid Chromatography With Tandem Mass Spectrometry (LC-MS/MS)}

LC-MS/MS is an analytical chemistry technique that includes physical separation of the analytes in liquid samples and followed by their mass-based identification (27). This technology contributed to the determination of the accurate mass, putative

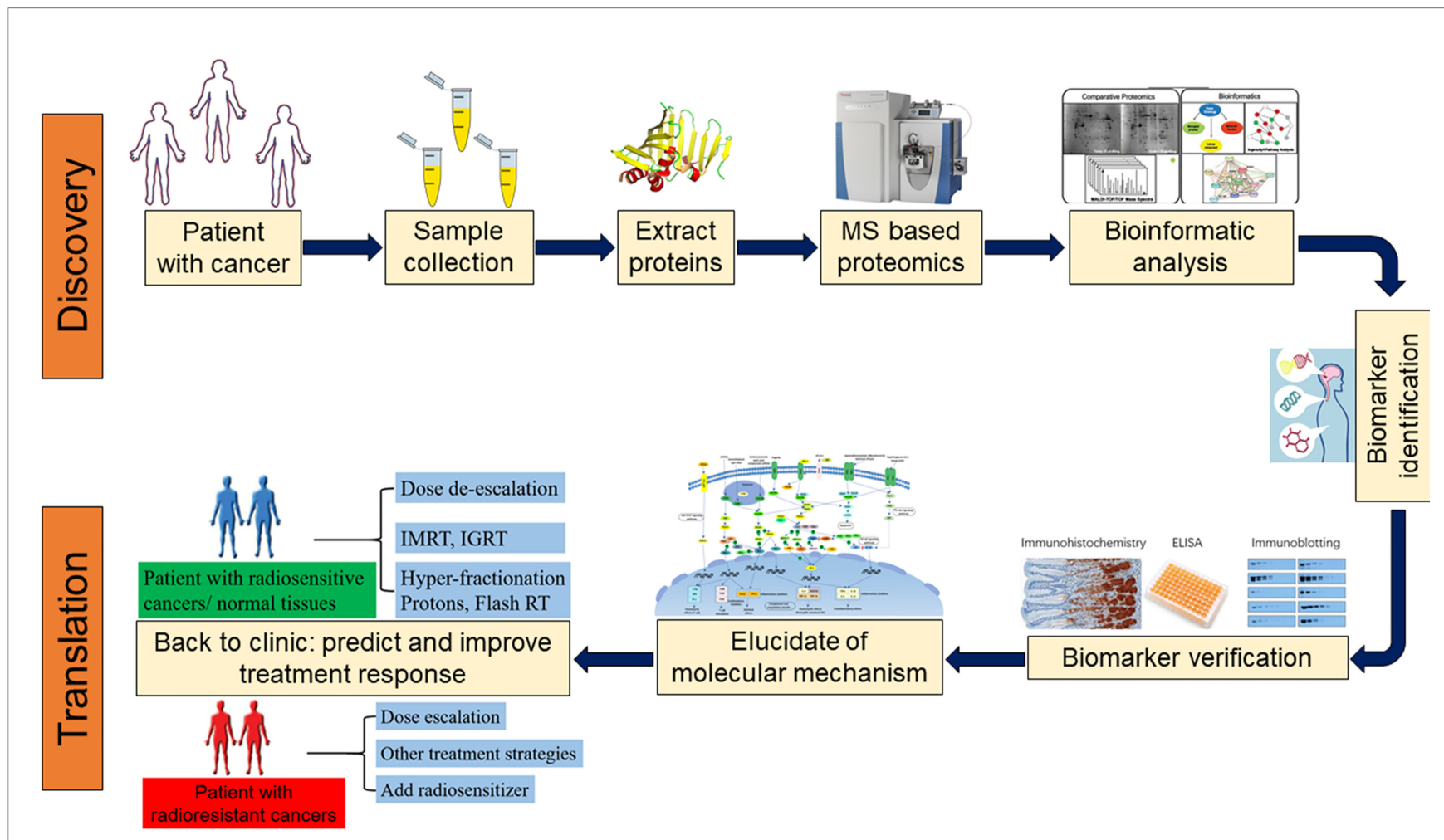

FIGURE 1 | Proteomics approaches for the discovery, validation, and clinical application of biomarkers. (MS, mass spectrometer; IGRT, Image-guided radiation therapy; IMRT, Intensity-modulated radiation therapy). 


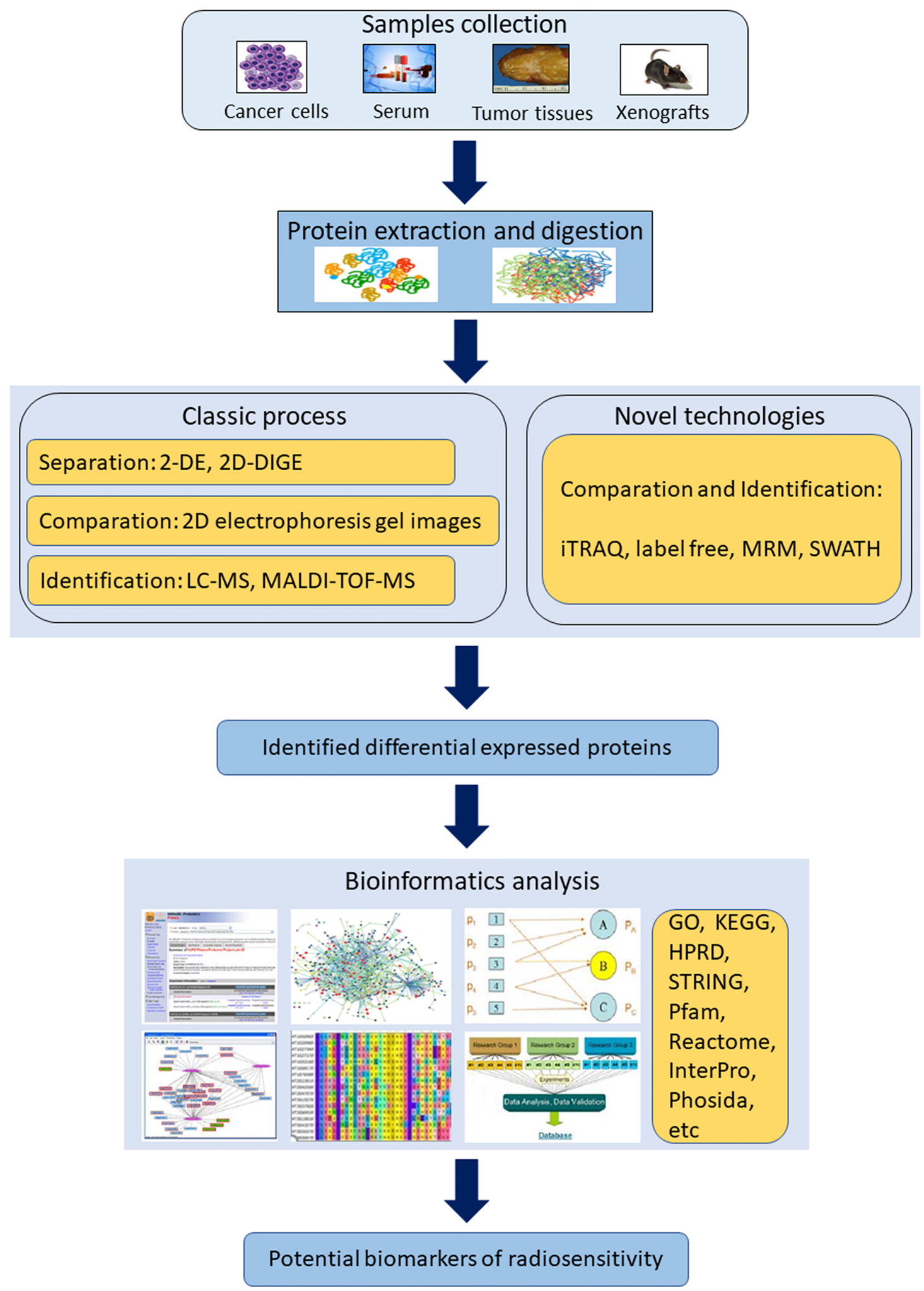

FIGURE 2 | Scheme of the workflow and methodological strategies for proteomics application in identifying radiosensitivity biomarkers. (2-DE, two-dimensional gel electrophoresis; 2D-DIGE, two-dimensional difference gel electrophoresis; GO, The Gene Ontology; HPRD, human protein reference database; iTRAQ, isobaric tags for relative and absolute quantitation; KEGG, Kyoto Encyclopedia of Genes and Genomes; MALDI-MS, matrix-assisted laser desorption ionization mass spectrometer; MRM, multiple reaction monitoring; SWATH, sequential window acquisition of all theoretical fragment ion spectra). 
formula, and elucidate the structure of the analyte with relatively small amounts of samples. Therefore, it has been widely used for the analysis of both small molecules and large protein molecules. Nevertheless, the instruments are extremely costly. Sample preparation is important for the extraction of target compounds from complex samples such as serum; otherwise, the system may be contaminated and resulted in a decreased sensitivity (28). Additionally, it is time consuming because of the large amount of information acquired from LC-MS/MS.

\subsection{Multiple Reaction Monitoring (MRM)}

MRM, also known as Selective Reaction Monitoring (SRM) is a technique based on triple quadrupole mass spectrometer and use the signal of selected tandem mass spectrometry fragment ions for further quantification (29). This method can eliminate most of the non-target detection and improve the detection sensitivity of the target proteins in complex samples, the results are reproducible. Meanwhile, MRM could be used for biomarker validation and has the potential to serve as a complementary tool to traditional methods including western blot, immunohistochemistry, and enzyme-linked immunosorbent assay (30). Despite these advantages, MRM may not suitable for proteins that are too short and variable, such as immunoglobulin. For the selected peptides from same target proteins, were observed, incomplete trypsin digestion of parental ions may lead to different quantitation outcomes (31).

\subsection{Sequential Window Acquisition of All Theoretical Mass Spectra (SWATH-MS)}

SWATH-MS is an emerging proteomic platform based on data independent acquisition (DIA) principle for label free quantification (32). It provides a complete and permanent information of all fragment ions in a sample, moreover, a spectral library is necessary to analysis the SWATH-MS data. This technology allows quantitative analysis of peptides covering a great amount of proteins with a high quantitative accuracy and reproducibility. The current disadvantage of SWATH-MS is that the peptide quantification is still less sensitive compared with MRM (33). SWATH-MS is a promising technique and novel algorithms are needed for identifying cancer radiosensitivity factors.

\section{RADIOSENSITIVITY BIOMARKERS IN CANCERS}

Approximately 50\% of tumors need to be treated with radiation therapy, and how to select eligible patients to receive radiotherapy was really a problem (34). Proteins expression were altered during radiotherapy, the application of proteomics to analyze these differential proteins has the potential to identify potential biomarkers of radiation resistance and further revealing the underlying molecular mechanisms. This will contribute to screen patients that eligible for radiotherapy and reveal novel targets of anticancer therapy. In the current study, the following terms were utilized: "neoplasms", "proteomic", "radiotherapy", we also manually checking reference lists to identify additional relevant studies.

\subsection{Head and Neck Cancers}

Head and neck cancers were the sixth leading cancer by incidence and the eighth major cause of cancer related death across the world, and squamous cell carcinoma was the most common type (35). Intensity-modulated radiotherapy was one of the most effective treatment strategies for head and neck cancers (36). Despite a good survival rates of $40 \%-50 \%$, some patients still exhibit resistance to radiotherapy and resulted in tumor recurrence (37). Identification of biomarkers related to head and neck cancer radiation resistance would helpful to improve patient survival.

\subsubsection{Nasopharyngeal Carcinoma}

Radiotherapy with or without chemotherapy is the standard treatment option for nasopharyngeal cancer because of the complex anatomical location of this disease (38). Radiation resistance is a severe obstacle to the successful treatment of nasopharyngeal carcinoma, and biomarkers related to the radiation response is of great importance (39). Wu et al. analyzed the proteomic changes of tumor tissues before and after radiation therapy in patients of nasopharyngeal carcinoma and found that endoplasmic reticulum protein 29 (ERP29), manganese superoxide dismutase (Mn-SOD), heat shock protein 27 (HSP27), and glutathione S-transferase (GST) were significantly upregulated and correlated with radiation resistance; ERp29 was significantly overexpressed in radiationresistant tumor tissues as verified by immunohistochemistry; the application of small RNA silencing technique to downregulate ERP29 expression enhanced both radiosensitivity and apoptosis in CNE-1 and 6-10B cells (40). This study suggests that ERP29 has the potential to be used as a marker for predicting the efficacy of radiotherapy in nasopharyngeal carcinoma. HSP27 was a chaperone belonged to the small heat shock protein family, the protein involved in the inhibition of apoptotic cell death, regulation of cell proliferation and differentiation (41). The analysis by Zhang et al. also confirmed HSP27 as a biomarker of radiation resistance in nasopharyngeal cancer (42). Zhang et al. performed serum proteins profiles using LC-MS/MS and found secreted protein acidic and cysteine rich (SPARC), serpin family D member $1 \mathrm{~S}$ (ERPIND1), complement C4B, peptidylprolyl Isomerase $\mathrm{B}$ (PPIB), and family with sequence similarity 173-member A (FAM173A) were associated with radiation resistance (43). To date, the role of SPARC, SERPIND1, and FAM173A in cancer remains unclear. Complement $\mathrm{C} 4 \mathrm{~B}$ was a component of the classical activation pathway, dysregulated complement expression within the tumor microenvironment was capable of suppressing antitumor immunity and resulted in poor clinical outcomes (44). PPIB was a cyclosporine-binding protein that participated to regulate cyclosporine A-mediated immunosuppression, further 
investigation revealed PPIB conferred radiation resistance in head and neck cancer via facilitating DNA repair (45).

In addition, Feng et al. established a radiation-resistant cell line CNE2-RR from fractionated radiotherapy irradiated nasopharyngeal carcinoma CNE2 cell line, and proteomic analysis identified 34 cases of differential proteins; after validated by 90 clinical tissue specimens of nasopharyngeal carcinoma, they identified that $14-3-3 \sigma$ and maspin were significantly downregulated, conversely, GRP78 and Mn-SOD were significantly upregulated; cellular studies confirmed that upregulation of 14-3-3 $\sigma$ could effectively improve the radiation sensitivity of CNE2-RR cell lines (46). GRP78 was a multifunctional protein folding chaperone in endoplasmic reticulum (ER), overexpression of GRP78 was associated with poor survival; suppressing GRP78 enhanced the efficacy of radiation therapy in cancer models (47). Li et al. compared CNE2-RR cell lines with CNE2 cell lines with MALDI-TOF-MS and uncovered 16 cases of proteins related to radiation sensitivity in CNE2-RR cell lines; among them, $\mathrm{Nm} 23 \mathrm{H} 1$ was significantly overexpressed and annexin A3 was significantly downregulated; however, the final results were not validated (48). With the same cell lines, another study reported fibrillin-2, CD166, sulfhydryl oxidase 1 and cofilin-2 as biomarkers of radiosensitivity using iTRAQ technique, further analysis revealed the 4 proteins involved in cell adhesion, migration and invasion (49). Similarly, Li et al. analyzed the CNE2-RR cell lines using LCMS/MS and found MAPK15 was involved in radiation resistance, the potential mechanisms were MAPK15 attenuated reactive oxygen species accumulation and promoted DNA damage repair (39). Accordingly, the above studies suggested that the results of different proteomic techniques were not identical, and further analyses were required in improving the homogeneity of these proteomic techniques.

\subsubsection{Other Types of Head and Neck Cancers}

Comparative analysis of protein profiles of radiation resistant cell lines QL1, SCC15 and SCC25 verified that Non-metastatic protein $23 \mathrm{H} 1(\mathrm{Nm} 23-\mathrm{H} 1)$ was highly expressed and could be used as a marker of radiation resistance (50). Nm23-H1 served as a housekeeping enzyme and involved in suppressing cancer cell metastasis, the activation of $\mathrm{Nm} 23-\mathrm{H} 1$ was capable of inhibiting cancer metastasis via redox regulation (51). Comparative proteomic analysis of head and neck squamous carcinoma cell lines $\mathrm{FaDu}$ and SCC25 with radiation-resistant cell lines FaDuRR and SCC25-RR showed that Ras-related C3 botulinum toxin substrate 1 ( $R a c 1)$ could be used as a new therapeutic target (52). Rac1 is a cytoskeleton involved in cell adhension, morphology, and movement; overexpression of Racl is associated with unfavorable survival in different type of cancer (53).

Interestingly, Matsukawa et al. performed a proteomic analysis of tumor tissues from 18 patients with locally advanced oral squamous carcinoma treated with neoadjuvant radiotherapy, galactose lectin 7 was identified and validated as a predictor of radiation resistance with a sensitivity of $96 \%$, a specificity of $39.5 \%$, in the meantime, the 5-year disease-specific survival rate was $75.2 \%$ in the galactose lectin 7 low expression group and $100 \%$ for the high expression group (54). Further analysis also confirmed that the invasive ability was decreased in galactose lectin 7 overexpressed cells; however, the invasive ability was not significantly altered after knockdown of galactose lectin 7 . Therefore, further investigation was necessary for galactose lectin 7 in predicting radiation resistance. Lin et al. analyzed three head and neck tumor cell lines, KB, SAS, and OECM1, and found that five proteins including Heat shock protein 96 (Gp96), Glucose regulatory protein 78 (GRP78), Heat shock protein 60 (HSP60), Ras-related protein Rab-40B (Rab40B) and Growth/differentiation factor-15 (GDF-15) was upregulated, while membrane-linked protein $\mathrm{V}$ was downregulation and was associated with radiation resistance; moreover, after silencing of Gp96, tumor cell growth was stunted, clonogenesis was diminished, and the proportion of cells in G2/M phase was increased; in the Gp96-silenced mouse transplant tumor model, the tumor size was significantly reduced when compared with normal mouse after receiving radiotherapy (55). Most of the biomarkers discovered in Lin et al's study were involved in the regulation of ER stress, and ER stress has been depicted as a hallmark of cancer (56). Our previous analysis also indicated ER stress is associated with radiation resistance (57). Proteomic analysis of the laryngeal cancer cell line HEp-2 and the radiation-resistant cell line HEp-2-RR showed that Chloride Intracellular Channel 1 (CLIC1) induces radiation resistance by inhibiting the production of reactive oxygen species (58). These studies suggest that a number of proteins were involved in the regulation of radiation sensitivity and can be used to predict radiotherapy efficacy and facilitate the development of novel strategies in anticancer therapy.

\subsection{Thoracic Cancers}

In recent years, the incidence of thoracic cancers including esophageal cancer, breast cancer, and lung cancer, were gradually increased across the world (59). Radiotherapy plays a key role in thoracic cancer patients that were not suitable for surgery or early stage non-small cell lung cancer, however, radiation resistance was a common cause of treatment failure $(60,61)$. Thereby, it was important to identify biomarkers of radiation resistance for each type of chest tumors.

\subsubsection{Esophageal Cancer}

With the application of two-dimensional protein liquid chromatography system and linear trap quadruple mass spectrometer, Cui et al. analyzed the serum proteomic profiles before and after radiotherapy of Kazakh esophageal cancer patients in Xinjiang and found 21 significant different expressed protein spots peaks; Clusterin (CLU) was detected as a radiation resistant biomarker, and Apolipoprotein A1 (APOA1) was discovered as a serum biomarker of esophageal squamous cell carcinoma (62). Based on next-generation MS, Maher et al. analyzed plasma protein expression changes before, during, and after radiotherapy in 31 esophageal cancer patients treated with neoadjuvant radiotherapy, final results indicated that complements $\mathrm{C} 3 \mathrm{a}$ and $\mathrm{C} 4 \mathrm{a}$ could be utilized in predicting the efficacy of neoadjuvant radiotherapy with a sensitivity of $78.6 \%$ and a specificity of $83.3 \%$ (21). Since then, the molecular 
mechanisms of allergenic toxins in predicting radiotherapy efficacy have been elaborated. Surace et al. found that radiotherapy induced tumor cell death, activated the complement system, and produced complement C3a and C5a, both $\mathrm{C} 3 \mathrm{a}$ and $\mathrm{C} 5 \mathrm{a}$ were concentrated in tumor tissue in the short term and induced specific anti-tumor immune responses; besides, glucocorticoids suppressed complement activation and decreased the efficacy of radiotherapy, therefore, these drugs should be administered with caution in cancer patients during radiotherapy (63). The study further confirmed that the application of immunosuppressants, such as dexamethasone, would lead to reduced treatment efficacy during radiotherapy. Proteomic analysis of esophageal cancer reveals a close relationship between radiotherapy and anti-tumor immune response, which may provide novel ideas for clinical studies of radiotherapy and immune checkpoint inhibitors.

\subsubsection{Breast Cancer}

Yang et al. used X-ray to treat breast cancer MDA- MB-231 cell line, and eight differential proteins were identified by MS as the following: Heat shock protein 70 (HSP70), Inosine Monophosphate Dehydrogenase 2 (IMPDH2), Eukaryotic Translation Initiation Factor 4H (EIF4H), Glyceraldehyde 3phosphate dehydrogenase (GAPDH), Vimentin (VIM), Tubulin Alpha 1b (TUBA1B), Tubulin Alpha 8 (TUBA8) and GRP78 (64). Kim et al. investigated protein expression profiles of breast cancer MDA- MB-231 BC cell treated with single or fractionated radiotherapy and found that several tumor-derived factors including CTSD (Cathepsin D), Gelsolin (GSN), and Mannose Receptor C Type 2 (MRC2) were upregulated, the results also suggested that these factors were involved in regulating the immune response during radiotherapy (65). These screened proteins may be involved in regulating breast cancer cell radiosensitivity and further studies were needed. Comparative proteomic analysis of the breast cancer radiation-sensitive cell line MCF+FIS4 and the radiation-resistant cell line MCF+FIR3 revealed that peroxidase II (PrxII) was significantly increased in the MCF+FIR3 cell line, and silencing of PrxII increased the radiation sensitivity of breast cancer cells, conversely, overexpressing PrxII in breast cancer cells resulted in radiation resistance; further exploration showed that reactive oxygen species were essential for breast cancer radiation resistance and stress-induced overexpression of PrxII protects breast cancer cells from radiation-induced oxidative damage and thus attenuates radiation sensitivity (66). Serum based LC-ESI-MS/ MS analysis confirmed oxidative stress response, acute phase response, and coagulation properties were hallmarks of radiosensitivity in breast cancer (67). However, it was worth noticing that the key molecular pathways involved in radiosensitivity is somehow influenced by the radiation doses (68). Guo et al. analyzed the protein kinase profiles of MCF-7 cell line and its cognate radiation-resistant cell line MCF-7/C6 and validated the results showed that checkpoint kinase 1 (CHK1), cell cycle protein-dependent kinases 1 and 2 (CDK1 and CDK2) were significantly upregulated in the radiation resistant cell lines; this suggests that both DNA repair and cell cycle were involved in regulating breast cancer radiation resistance (69).
To detect the homogeneity and heterogeneity between different proteomics techniques, Laura et al. analyzed breast cancer radiation resistant cell lines (MCF7-RR, MDA-MB-231$\mathrm{RR}$ and $\mathrm{T} 47 \mathrm{D}-\mathrm{RR}$ ) with their cognate radiation therapy sensitive cell lines using three different proteomics techniques: the twodimensional gel electrophoresis in combination with MS, liquid chromatography coupled with tandem mass spectrometry, and quantitative proteomics using iTRAQ technique; the resules verified that $26 \mathrm{~S}$ proteasome and GRP78 were significantly down-regulated in all radiation-resistant cell lines, in addition, very few overlapping proteins were identified by the three techniques, which indicating the different advantages with different proteomics techniques (70). Over the past decades, GRP78 has been recognized as a target in anticancer strategies (71). Several compounds and antibodies targeting GRP78 have been investigated in various type of cancers $(47,72,73)$.

Although the aforementioned studies uncovered several potential radiation-resistant biomarkers, their clinical significance has not been well investigated. Pietrowska et al. analyzed the protein expression profiles of plasmas from breast cancer patients treated with surgery and adjuvant radiotherapy, in the surgery alone group, there was insignificant changes in protein expression between preoperative and postoperative serum samples; in patients receiving adjuvant radiotherapy, they detected two serum peptides (registered $\mathrm{m} / \mathrm{z}$ values 2,184 and 5,403 Da) were significantly downregulated (74). This study confirmed that radiotherapy can modulating protein expression in the plasma of cancer patients, however, further bioinformatics analysis was needed to identify potential biomarkers of radiation resistance.

\subsubsection{Non-Small Cell Lung Cancer}

Yun et al. established a radiation resistant H460 (RR-H460) cell line that displayed cancer stem like cell phenotypes from parental radiosensitive $\mathrm{H} 460$ non-small cell lung cancer cells, proteomic analyses of the two cell lines by LC-MS/MS revealed VIM, GRP78, fatty acid synthase (FASN), ubiquinol-cytochrome $\mathrm{C}$ reductase complex core protein 1 (UQCRC1), plasminogen activator inhibitor 2 (PAI-2), nodal modulator 2 (NOMO2), kinesin light chain 4 (KLC4) and procollagen-lysine 2-oxoglutarate 5dioxygenase 3 (PLOD3) as radiation resistance biomarkers. Among these identified proteins, VIM, GRP78, FASN, UQCRC1 have been validated in the prediction of radiation resistance (75). Indeed, GRP78 and VIM have been depicted as radiation resistance factors in other type of tumors $(46,65)$. Walker et al. collected plasma from patients with locally advanced non-small cell lung cancer treated with radical radiotherapy, proteomic analysis revealed that C-reactive protein (CRP), Lipopolysaccharide Binding Protein (LBP), and Leucine Rich Alpha-2-Glycoprotein 1 (LRG1) were significantly altered during radiotherapy, in the squamous lung cancer subgroup, both CRP and LRG1 were significantly elevated in patients with survival $<17$ months (22). CRP is a predominant protein of the acute phase response; this protein involved in inflammation response and the developing of pro-metastatic tumor microenvironment (76). LRG1 promotes neovascularization via regulating TGF- $\beta$ signaling (77). Radiotherapy induces the expression and release of pro- 
angiogenic factors, and the combination of LRG1 inhibitors might be a promising strategy in improving the efficacy of radiotherapy and patients' prognosis (22). A proteomic analysis of non-small cell lung cancer conducted by Huang et al. identified six potential biomarkers in the radiation-resistant group, the results suggested that $\alpha 1$ antitrypsin could be used as a marker of radiation resistance (78). In conclusion, the above analyses suggested that the screening of protentional biomarkers before and after radiotherapy using proteomics technology can not only be used to predict radiotherapy efficacy and patient survival, but also contribute to the design of novel anti-tumor agents.

\subsection{Abdominopelvic Tumors}

Abdominopelvic tumors account for more than half of tumors in the human body, and radiotherapy was extremely valuable in the comprehensive treatment of abdominopelvic tumors. Nevertheless, the existence of radiation resistance lead to cancer recurrence or progression. Screening for biomarkers of radiation resistance was useful in stratifying patients with abdominopelvic tumors and optimal treatment strategies can be conducted.

\subsubsection{Rectal Cancer}

The protein profiles of rectal cancer tissue of patients before and after radiotherapy were analyzed by MALDI-TOF-MS, both ERp57and Galectin-3 were the main biomarkers identified (79). ERp57 belonged to the protein disulfide isomerase family, this protein conferred immune escape via multiple immune regulatory processes (80). Galectin-3 was a member of the lectin family and contributed to tumor microenvironment immunosuppression and immune cell regulation (81). Besides, Chauvin et al. analyzed the protein expression profile of 23 rectal cancer patients treated with neoadjuvant radiotherapy and found that Interferon Induced Protein with Tetratricopeptide Repeats 1 (IFIT1), FAST Kinase Domains 2 (FASTKD2), Phosphatidylinositol-5-Phosphate 4-Kinase Type 2 Beta (PIP4K2B), AT-Rich Interaction Domain 1B (ARID1B), and Solute Carrier Family 25 Member 33 (SLC25A33) were overexpressed in patients achieved complete remission, in the meantime, Caldesmon 1 (CALD1), Carboxypeptidase A3 (CPA3), Beta-1,3-Galactosyltransferase 5 (B3GALT5), Receptor Interacting Serine/Threonine Kinase 1 (RIPK1), and CD117 were upregulated in patients with poor response (82). Among these identified biomarkers, PIP4K2B was a lipid kinase and involved in the regulation of cancer cell proliferation via transduced changes in GTP into changes in the levels of the stress regulated phosphoinositide PtdIns5P (83). Furthermore, CALD1, a biomarker of cancer associated fibroblasts, was found to be an independent biomarker of poor survival by gene expression profiles of micro-dissected rectal cancer (84). To date, the mechanisms of these altered proteins mediate radiation resistance in rectal tumor cells have not been fully elucidated, and large sample size clinical studies were required. Additionally, it should be noted that the changes of radiosensitivity biomarkers in serum may be tumor stages specific (81).

\subsubsection{Prostate Cancer}

Skvortsova et al. compared the protein profiles between three prostate cancer radiation therapy-resistant cell lines (PC3-RR,
DU145-RR, and LNCaP-RR) and their cognate radiation sensitive cell lines, the result revealed five proteins were significantly altered: NME/NM23 Nucleoside Diphosphate Kinase 1 (NME1), Heat Shock Protein Family A Member 8 (HSPA8), Apurinic/Apyrimidinic Endodeoxyribonuclease 1 (APEX1), and Ras-related protein Rab-11A (RAB11A); APEX1 was an DNA repair enzyme, knockout of APEX1 significantly enhanced radiosensitivity of prostate cancer cells (85). It was worth noting that NME1 served as a metastasis suppressor in cancer cells, this enzyme decreased pEGFR and pAkt expression in a dynamin dependent manner and contributed to metastasis suppression via altering tumor endocytic and motility phenotypes (86). Chang et al. analyzed the protein profiles of three prostate cancer radiation-resistant cell lines (PC3, DU145, and LNCaP) and their cognate radiation sensitive cell lines using label-free liquid chromatography tandem mass spectrometry, final results revealed Aldolase, Fructose-Bisphosphate A (ALDOA) involved in the regulation of radiation sensitivity both on prostate cancer cell lines and its transplant tumor models (87). Both aldolase and ALDOA involved in glucose metabolism, these enzymes promoted cancer cell metastasis via interacting with $\gamma$-actin (88). One of the glycolysis markers, lactate dehydrogenase $\mathrm{A}$ has been demonstrated as a biomarker of radiation resistance in a radioresistant prostate cancer xenograft mouse model using LC-MS/MS (89). Glycolysis was associated with treatment resistance in different type of cancers, thereby serving as a promising target of antitumor therapy (90). These studies suggested that the mechanisms of radiation resistance regulation were really complex and the combination of various proteomic techniques can be used to uncover novel radiation resistance biomarkers.

\subsection{Extracranial Tumors in Children}

Neuroblastoma was the most common extracranial solid tumor in children, with approximately $60 \%$ of children experiencing disease progression and metastasis (91). Abou-Antoun et al. compared the protein expression profiles of anchorage dependent and anchorage independent murine neuroblastoma and found that a dose of $2.5 \mathrm{~Gy}$ irradiation induced overexpression of survivin, Cyclin-dependent kinase 1 (CDC2), and Poly(ADP-Ribose) Polymerase 1 (PARP1) in unanchored neuroblastoma, and knockdown of survivin combined with multitargeted receptor tyrosine kinase inhibitor sunitinib enhanced the radiosensitivity of anchorage independent neuroblastoma; Anchorage-independent neuroblastoma has a different protein expression profile when compared with anchorage-dependent tumors and thus exhibits a higher degree of malignant phenotype and much more resistance to radiotherapy (92). CDC2 played a crucial role in regulating the transition of cells from $\mathrm{G} 2$ phase to mitotic $\mathrm{M}$ phase, targeting CDC2 lead to cell cycle arrest at the G2 phase (93). Additionally, PARP1 was a common nuclear protein and played an essential role in the stabilization of DNA replication forks; suppressing PARP1 was a complementary to conventional therapy by broken DNA repair mechanisms (94). Overall, these results suggested that 


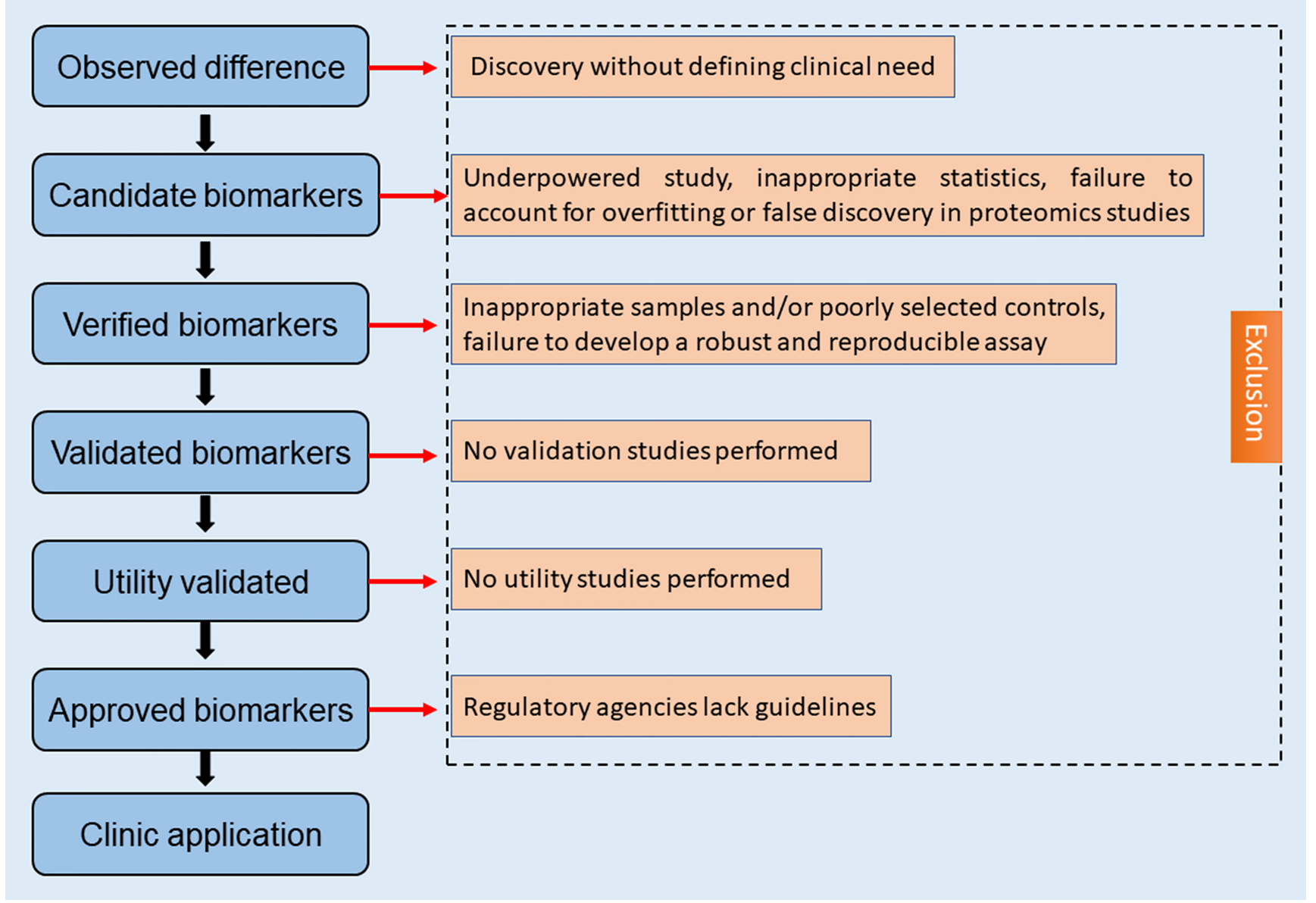

FIGURE 3 | Radiosensitivity biomarkers development from proteomics to clinical.

inhibition of these altered proteins and their related signaling pathways has the potential to improve radiosensitivity of extracranial tumors.

\section{CHALLENGES OF PROTEOMICS}

Although proteomics focusing on the expression altered proteins and their activities in cells and organisms, this technology was still in its initial stage. There were many problems to be solved: firstly, sample preparing process, there was no uniform experimental conditions yet secondly, although a large number of proteins related to radiosensitivity have been discovered using proteomics techniques, more effective methods were required to identify the most specific targets (95). Moreover, the current proteomics analysis was expensive, which limits the possibility of further expanding the sample size; the detailed molecular mechanisms of screened biomarkers have not been well clarified; lastly, there are a great amount of work need to do for the application of radiosensitivity biomarkers that developed from proteomics to clinical (Figure 3). It was believed that with the application of new technologies, large-scale proteomics analysis will become possible.

\section{SUMMARY AND PERSPECTIVE}

The use of proteomics to analyze radiation sensitivity biomarkers in tissues and plasma of cancer patients treated with radiotherapy has shown great promise and could clear some of the obstacles in precision tumor therapy. With the development of high-precision mass spectrometer, comprehensive analysis software and multimodel database, it was possible to comprehensively analysis the mechanisms underlying radiation resistance. This was helpful in the screening of patients appropriate for receiving radiotherapy in clinical practice, and to design novel targeted agents based on the discovered biomarkers, thus guiding the implementation of precision radiotherapy.

\section{AUTHOR CONTRIBUTIONS}

HL and HG contributed equally to the study. All authors contributed to the article and approved the submitted version. 


\section{REFERENCES}

1. Chandra RA, Keane FK, Voncken FEM, Thomas CRJr. Contemporary Radiotherapy: Present and Future. Lancet (2021) 398:171-84. doi: 10.1016/ S0140-6736(21)00233-6

2. Tsukamoto S, Kido A, Tanaka Y, Facchini G, Peta G, Rossi G, et al. Current Overview of Treatment for Metastatic Bone Disease. Curr Oncol (2021) 28:3347-72. doi: 10.3390/curroncol28050290

3. Lee J, Byun HK, Koom WS, Lee YC, Seong J. Efficacy of Radiotherapy for Gastric Bleeding Associated With Advanced Gastric Cancer. Radiat Oncol (2021) 16:161. doi: 10.1186/s13014-021-01884-5

4. Straka C, Ying J, Kong FM, Willey CD, Kaminski J, Kim DW. Review of Evolving Etiologies, Implications and Treatment Strategies for the Superior Vena Cava Syndrome. Springerplus (2016) 5:229. doi: 10.1186/s40064-016-1900-7

5. Rycaj K, Tang DG. Cancer Stem Cells and Radioresistance. Int J Radiat Biol (2014) 90:615-21. doi: 10.3109/09553002.2014.892227

6. Ho D, Quake SR, McCabe ERB, Chng WJ, Chow EK, Ding X, et al. Enabling Technologies for Personalized and Precision Medicine. Trends Biotechnol (2020) 38:497-518. doi: 10.1016/j.tibtech.2019.12.021

7. Lehman SL, Wilson ED, Camphausen K, Tofilon PJ. Translation Initiation Machinery as a Tumor Selective Target for Radiosensitization. Int J Mol Sci (2021) 22:10664. doi: 10.3390/ijms221910664

8. Luo H, Wang X, Wang Y, Dan Q, Ge H. Mannose Enhances the RadioSensitivity of Esophageal Squamous Cell Carcinoma With Low MPI Expression by Suppressing Glycolysis. Discov Oncol (2022) 13:1. doi: 10.1007/s12672-021-00447-0

9. Olivier M, Asmis R, Hawkins GA, Howard TD, Cox LA. The Need for MultiOmics Biomarker Signatures in Precision Medicine. Int J Mol Sci (2019) 20:4781. doi: 10.3390/ijms20194781

10. Castillo-Tandazo W, Mutsaers AJ, Walkley CR. Osteosarcoma in the Post Genome Era: Preclinical Models and Approaches to Identify Tractable Therapeutic Targets. Curr Osteoporos Rep (2019) 17:343-52. doi: 10.1007/s11914-019-00534-w

11. Jalili M, Gebhardt T, Wolkenhauer O, Salehzadeh-Yazdi A. Unveiling Network-Based Functional Features Through Integration of Gene Expression Into Protein Networks. Biochim Biophys Acta Mol Basis Dis (2018) 1864:2349-59. doi: 10.1016/j.bbadis.2018.02.010

12. Buccitelli C, Selbach M. mRNAs, Proteins and the Emerging Principles of Gene Expression Control. Nat Rev Genet (2020) 21:630-44. doi: 10.1038/ s41576-020-0258-4

13. Doll S, Gnad F, Mann M. The Case for Proteomics and Phospho-Proteomics in Personalized Cancer Medicine. Proteomics Clin Appl (2019) 13:e1800113. doi: 10.1002/prca.201800113

14. Huang R, Chen Z, He L, He N, Xi Z, Li Z, et al. Mass Spectrometry-Assisted Gel-Based Proteomics in Cancer Biomarker Discovery: Approaches and Application. Theranostics (2017) 7:3559-72. doi: 10.7150/thno.20797

15. Smith R, Wang J, Seymour C, Fernandez-Palomo C, Fazzari J, Schultke E, et al. Homogenous and Microbeam X-Ray Radiation Induces Proteomic Changes in the Brains of Irradiated Rats and in the Brains of Nonirradiated Cage Mate Rats. Dose Response (2018) 16:1559325817750068. doi: 10.1177/ 1559325817750068

16. Mardamshina M, Geiger T. Next-Generation Proteomics and Its Application to Clinical Breast Cancer Research. Am J Pathol (2017) 187:2175-84. doi: 10.1016/j.ajpath.2017.07.003

17. Ternette N, Olde Nordkamp MJM, Muller J, Anderson AP, Nicastri A, Hill AVS, et al. Immunopeptidomic Profiling of HLA-A2-Positive Triple Negative Breast Cancer Identifies Potential Immunotherapy Target Antigens. Proteomics (2018) 18:e1700465. doi: 10.1002/pmic.201700465

18. Silvestri GA, Tanner NT, Kearney P, Vachani A, Massion PP, Porter A, et al. Assessment of Plasma Proteomics Biomarker's Ability to Distinguish Benign From Malignant Lung Nodules: Results of the PANOPTIC (Pulmonary Nodule Plasma Proteomic Classifier) Trial. Chest (2018) 154:491-500. doi: 10.1016/j.chest.2018.02.012

19. Latosinska A, Frantzi M, Vlahou A, Merseburger AS, Mischak H. Clinical Proteomics for Precision Medicine: The Bladder Cancer Case. Proteomics Clin Appl (2018) 12:1700074. doi: 10.1002/prca.201700074

20. Sun BB, Maranville JC, Peters JE, Stacey D, Staley JR, Blackshaw J, et al. Genomic Atlas of the Human Plasma Proteome. Nature (2018) 558:73-9. doi: $10.1038 /$ s41586-018-0175-2
21. Maher SG, McDowell DT, Collins BC, Muldoon C, Gallagher WM, Reynolds JV. Serum Proteomic Profiling Reveals That Pretreatment Complement Protein Levels Are Predictive of Esophageal Cancer Patient Response to Neoadjuvant Chemoradiation. Ann Surg (2011) 254:809-16; discussion 8167. doi: 10.1097/SLA.0b013e31823699f2

22. Walker MJ, Zhou C, Backen A, Pernemalm M, Williamson AJ, Priest LJ, et al. Discovery and Validation of Predictive Biomarkers of Survival for Non-Small Cell Lung Cancer Patients Undergoing Radical Radiotherapy: Two Proteins With Predictive Value. EBioMedicine (2015) 2:841-50. doi: 10.1016/ j.ebiom.2015.06.013

23. Giudice G, Petsalaki E. Proteomics and Phosphoproteomics in Precision Medicine: Applications and Challenges. Brief Bioinform (2019) 20:767-77. doi: $10.1093 / \mathrm{bib} / \mathrm{bbx} 141$

24. Singhal N, Kumar M, Kanaujia PK, Virdi JS. MALDI-TOF Mass Spectrometry: An Emerging Technology for Microbial Identification and Diagnosis. Front Microbiol (2015) 6:791. doi: 10.3389/fmicb.2015.00791

25. Chen X, Sun Y, Zhang T, Shu L, Roepstorff P, Yang F. Quantitative Proteomics Using Isobaric Labeling: A Practical Guide. Genomics Proteomics Bioinf (2022) 22:1-8. doi: 10.1016/j.gpb.2021.08.012

26. Cai XZ, Zeng WQ, Xiang Y, Liu Y, Zhang HM, Li H, et al. iTRAQ-Based Quantitative Proteomic Analysis of Nasopharyngeal Carcinoma. J Cell Biochem (2015) 116:1431-41. doi: 10.1002/jcb.25105

27. Bach T, An G. Importance of Utilizing Natural Isotopologue Transitions in Expanding the Linear Dynamic Range of LC-MS/MS Assay for SmallMolecule Pharmacokinetic Sample Analysis - A Mini-Review. J Pharm Sci (2021) 21:690. doi: 10.1016/j.xphs.2021.12.012

28. Jickells S. Sample Preparation. In: Analytical Techniques in Forensic Science. New Jersey: Wiley (2021). doi: 10.1002/9781119373421.ch4

29. Mermelekas G, Vlahou A, Zoidakis J. SRM/MRM Targeted Proteomics as a Tool for Biomarker Validation and Absolute Quantification in Human Urine. Expert Rev Mol Diagn (2015) 15:1441-54. doi: 10.1586/14737159. 2015.1093937

30. Schaaij-Visser TB, Brakenhoff RH, Leemans CR, Heck AJ, Slijper M. Protein Biomarker Discovery for Head and Neck Cancer. J Proteomics (2010) 73:1790-803. doi: 10.1016/j.jprot.2010.01.013

31. You J, Willcox MD, Madigan MC, Wasinger V, Schiller B, Walsh BJ, et al. Chapter Four - Tear Fluid Protein Biomarkers. In: Makowski GS, editor. Advances in Clinical Chemistry. Amsterdam: Elsevier (2013). doi: 10.1016/ B978-0-12-800096-0.00004-4

32. Ludwig C, Gillet L, Rosenberger G, Amon S, Collins BC, Aebersold R. DataIndependent Acquisition-Based SWATH-MS for Quantitative Proteomics: A Tutorial. Mol Syst Biol (2018) 14:e8126. doi: 10.15252/msb.20178126

33. Schmidlin T, Garrigues L, Lane CS, Mulder TC, van Doorn S, Post H, et al. Assessment of SRM, MRM(3), and DIA for the Targeted Analysis of Phosphorylation Dynamics in Non-Small Cell Lung Cancer. Proteomics (2016) 16:2193-205. doi: 10.1002/pmic.201500453

34. Pinkawa M. Radiotherapy. Dtsch Arztebl Int (2018) 115:596. doi: 10.3238/ arztebl.2018.0596a

35. Siegel RL, Miller KD, Jemal A. Cancer Statistics, 2020. CA Cancer J Clin (2020) 70:7-30. doi: 10.3322/caac. 21590

36. Alterio D, Marvaso G, Ferrari A, Volpe S, Orecchia R, Jereczek-Fossa BA. Modern Radiotherapy for Head and Neck Cancer. Semin Oncol (2019) 46:233-45. doi: 10.1053/j.seminoncol.2019.07.002

37. Chow LQM. Head and Neck Cancer. N Engl J Med (2020) 382:60-72. doi: 10.1056/NEJMra1715715

38. Li F, Chen FP, Chen YP, Chen Y, He XJ, Huang XD, et al. Clinical Characteristics and Prognostic Factors of Early and Late Recurrence After Definitive Radiotherapy for Nasopharyngeal Carcinoma. Front Oncol (2020) 10:1469. doi: 10.3389/fonc.2020.01469

39. Li Z, Li N, Shen L, Fu J. Quantitative Proteomic Analysis Identifies MAPK15 as a Potential Regulator of Radioresistance in Nasopharyngeal Carcinoma Cells. Front Oncol (2018) 8:548. doi: 10.3389/fonc.2018.00548

40. Wu P, Zhang H, Qi L, Tang Q, Tang Y, Xie Z, et al. Identification of ERp29 as a Biomarker for Predicting Nasopharyngeal Carcinoma Response to Radiotherapy. Oncol Rep (2012) 27:987-94. doi: 10.3892/or.2011.1586

41. Katsogiannou M, Andrieu C, Rocchi P. Heat Shock Protein 27 Phosphorylation State Is Associated With Cancer Progression. Front Genet (2014) 5:346. doi: 10.3389/fgene.2014.00346 
42. Zhang B, Qu JQ, Xiao L, Yi H, Zhang PF, Li MY, et al. Identification of Heat Shock Protein 27 as a Radioresistance-Related Protein in Nasopharyngeal Carcinoma Cells. J Cancer Res Clin Oncol (2012) 138:2117-25. doi: 10.1007/ s00432-012-1293-0

43. Zhang G, Zhang K, Li C, Li Y, Li Z, Li N, et al. Serum Proteomics Identify Potential Biomarkers for Nasopharyngeal Carcinoma Sensitivity to Radiotherapy. Biosci Rep (2019) 39:BSR20190027. doi: 10.1042/BSR20190027

44. O'Brien RM, Cannon A, Reynolds JV, Lysaght J, Lynam-Lennon N. Complement in Tumourigenesis and the Response to Cancer Therapy. Cancers (Basel) (2021) 13:1209. doi: 10.3390/cancers13061209

45. Williams PD, Owens CR, Dziegielewski J, Moskaluk CA, Read PW, Larner JM, et al. Cyclophilin B Expression Is Associated With In Vitro Radioresistance and Clinical Outcome After Radiotherapy. Neoplasia (2011) 13:1122-31. doi: 10.1593/neo.111398

46. Feng XP, Yi H, Li MY, Li XH, Yi B, Zhang PF, et al. Identification of Biomarkers for Predicting Nasopharyngeal Carcinoma Response to Radiotherapy by Proteomics. Cancer Res (2010) 70:3450-62. doi: 10.1158/ 0008-5472.CAN-09-4099

47. Dadey DYA, Kapoor V, Hoye K, Khudanyan A, Collins A, Thotala D, et al. Antibody Targeting GRP78 Enhances the Efficacy of Radiation Therapy in Human Glioblastoma and Non-Small Cell Lung Cancer Cell Lines and Tumor Models. Clin Cancer Res (2017) 23:2556-64. doi: 10.1158/10780432.CCR-16-1935

48. Li L, Huang S, Zhu X, Zhou Z, Liu Y, Qu S, et al. Identification of Radioresistance-Associated Proteins in Human Nasopharyngeal Carcinoma Cell Lines by Proteomic Analysis. Cancer Biother Radiopharm (2013) 28:3804. doi: $10.1089 / \mathrm{cbr} .2012 .1348$

49. Chen ZT, Li L, Guo Y, Qu S, Zhao W, Chen H, et al. Analysis of the Differential Secretome of Nasopharyngeal Carcinoma Cell Lines CNE-2R and CNE-2. Oncol Rep (2015) 34:2477-88. doi: 10.3892/or.2015.4255

50. Lee SY, Park HR, Cho NH, Choi YP, Rha SY, Park SW, et al. Identifying Genes Related to Radiation Resistance in Oral Squamous Cell Carcinoma Cell Lines. Int J Oral Maxillofac Surg (2013) 42:169-76. doi: 10.1016/ j.ijom.2012.10.022

51. Kim B, Lee KJ. Activation of Nm23-H1 to Suppress Breast Cancer Metastasis via Redox Regulation. Exp Mol Med (2021) 53:346-57. doi: 10.1038/s12276021-00575-1

52. Skvortsov S, Jimenez CR, Knol JC, Eichberger P, Schiestl B, Debbage P, et al. Radioresistant Head and Neck Squamous Cell Carcinoma Cells: Intracellular Signaling, Putative Biomarkers for Tumor Recurrences and Possible Therapeutic Targets. Radiother Oncol (2011) 101:177-82. doi: 10.1016/ j.radonc.2011.05.067

53. Liang J, Oyang L, Rao S, Han Y, Luo X, Yi P, et al. Rac1, A Potential Target for Tumor Therapy. Front Oncol (2021) 11:674426. doi: 10.3389/ fonc.2021.674426

54. Matsukawa S, Morita K, Negishi A, Harada H, Nakajima Y, Shimamoto H, et al. Galectin-7 as a Potential Predictive Marker of Chemo- and/or RadioTherapy Resistance in Oral Squamous Cell Carcinoma. Cancer Med (2014) 3:349-61. doi: 10.1002/cam4.195

55. Lin TY, Chang JT, Wang HM, Chan SH, Chiu CC, Lin CY, et al. Proteomics of the Radioresistant Phenotype in Head-And-Neck Cancer: Gp96 as a Novel Prediction Marker and Sensitizing Target for Radiotherapy. Int J Radiat Oncol Biol Phys (2010) 78:246-56. doi: 10.1016/j.ijrobp.2010.03.002

56. Urra H, Dufey E, Avril T, Chevet E, Hetz C. Endoplasmic Reticulum Stress and the Hallmarks of Cancer. Trends Cancer (2016) 2:252-62. doi: 10.1016/ j.trecan.2016.03.007

57. Luo H, Song H, Mao R, Gao Q, Feng Z, Wang N, et al. Targeting ValosinContaining Protein Enhances the Efficacy of Radiation Therapy in Esophageal Squamous Cell Carcinoma. Cancer Sci (2019) 110:3464-75. doi: 10.1111/ cas. 14184

58. Kim JS, Chang JW, Yun HS, Yang KM, Hong EH, Kim DH, et al. Chloride Intracellular Channel 1 Identified Using Proteomic Analysis Plays an Important Role in the Radiosensitivity of HEp-2 Cells via Reactive Oxygen Species Production. Proteomics (2010) 10:2589-604. doi: 10.1002/ pmic. 200900523

59. Eloranta S, Smedby KE, Dickman PW, Andersson TM. Cancer Survival Statistics for Patients and Healthcare Professionals - A Tutorial of RealWorld Data Analysis. J Intern Med (2021) 289:12-28. doi: 10.1111/joim.13139
60. Baker S, Dahele M, Lagerwaard FJ, Senan S. A Critical Review of Recent Developments in Radiotherapy for Non-Small Cell Lung Cancer. Radiat Oncol (2016) 11:115. doi: 10.1186/s13014-016-0693-8

61. Luo H, Ge H, Cui Y, Zhang J, Fan R, Zheng A, et al. Systemic Inflammation Biomarkers Predict Survival in Patients of Early Stage Non-Small Cell Lung Cancer Treated With Stereotactic Ablative Radiotherapy - A Single Center Experience. J Cancer (2018) 9:182-8. doi: 10.7150/jca.21703

62. Li C, Xia G, Jianqing Z, Mei Y, Ge B, Li Z. Serum Differential Protein Identification of Xinjiang Kazakh Esophageal Cancer Patients Based on the Two-Dimensional Liquid-Phase Chromatography and LTQ MS. Mol Biol Rep (2014) 41:2893-905. doi: 10.1007/s11033-014-3145-2

63. Surace L, Lysenko V, Fontana AO, Cecconi V, Janssen H, Bicvic A, et al. Complement Is a Central Mediator of Radiotherapy-Induced Tumor-Specific Immunity and Clinical Response. Immunity (2015) 42:767-77. doi: 10.1016/ j.immuni.2015.03.009

64. Yang XR. Effects of X-Ray Radiation on the Biological Characteristics of Breast Cancer Cells MDA-MB-231 and Proteomic Research. Lanzhou: Doctoral Dissertation, Lanzhou: Lanzhou University (2016). doi: 10.7666/d.D01033563

65. Kim MH, Jung SY, Ahn J, Hwang SG, Woo HJ, An S, et al. Quantitative Proteomic Analysis of Single or Fractionated Radiation-Induced Proteins in Human Breast Cancer MDA-MB-231 Cells. Cell Biosci (2015) 5:2. doi: $10.1186 / 2045-3701-5-2$

66. Wang T, Tamae D, LeBon T, Shively JE, Yen Y, Li JJ. The Role of Peroxiredoxin II in Radiation-Resistant MCF-7 Breast Cancer Cells. Cancer Res (2005) 65:10338-46. doi: 10.1158/0008-5472.CAN-04-4614

67. Skiold S, Azimzadeh O, Merl-Pham J, Naslund I, Wersall P, Lidbrink E, et al. Unique Proteomic Signature for Radiation Sensitive Patients; a Comparative Study Between Normo-Sensitive and Radiation Sensitive Breast Cancer Patients. Mutat Res (2015) 776:128-35. doi: 10.1016/j.mrfmmm.2014.12.002

68. Shahani M, Shakeri J, Akbari ME, Arefnezhad B, Tafti A, Zali H, et al. Transcriptomic and Proteomic Approaches Reveal Biological Basis of Intraoperative Radiotherapy-Treated Tumor Bed Modification in Breast Cancer Patients: A Pilot Study. J Proteomics (2020) 212:103596. doi: 10.1016/ j.jprot.2019.103596

69. Guo L, Xiao Y, Fan M, Li JJ, Wang Y. Profiling Global Kinome Signatures of the Radioresistant MCF-7/C6 Breast Cancer Cells Using MRM-Based Targeted Proteomics. J Proteome Res (2015) 14:193-201. doi: 10.1021/pr500919w

70. Smith L, Qutob O, Watson MB, Beavis AW, Potts D, Welham KJ, et al. Proteomic Identification of Putative Biomarkers of Radiotherapy Resistance: A Possible Role for the 26S Proteasome? Neoplasia (2009) 11:1194-207. doi: 10.1593/neo.09902

71. Lu G, Luo H, Zhu X. Targeting the GRP78 Pathway for Cancer Therapy. Front Med (Lausanne) (2020) 7:351. doi: 10.3389/fmed.2020.00351

72. Qiao Y, Dsouza C, Matthews AA, Jin Y, He W, Bao J, et al. Discovery of Small Molecules Targeting GRP78 for Antiangiogenic and Anticancer Therapy. Eur J Med Chem (2020) 193:112228. doi: 10.1016/j.ejmech.2020.112228

73. Zheng Y, Liu P, Wang N, Wang S, Yang B, Li M, et al. Betulinic Acid Suppresses Breast Cancer Metastasis by Targeting GRP78-Mediated Glycolysis and ER Stress Apoptotic Pathway. Oxid Med Cell Longev (2019) 2019:8781690. doi: 10.1155/2019/8781690

74. Pietrowska M, Polanska J, Marczak L, Behrendt K, Nowicka E, Stobiecki M, et al. Mass Spectrometry-Based Analysis of Therapy-Related Changes in Serum Proteome Patterns of Patients With Early-Stage Breast Cancer. J Transl Med (2010) 8:66. doi: 10.1186/1479-5876-8-66

75. Yun HS, Baek JH, Yim JH, Um HD, Park JK, Song JY, et al. Radiotherapy Diagnostic Biomarkers in Radioresistant Human H460 Lung Cancer Stem-Like Cells. Cancer Biol Ther (2016) 17:208-18. doi: 10.1080/15384047.2016.1139232

76. Hart PC, Rajab IM, Alebraheem M, Potempa LA. C-Reactive Protein and Cancer-Diagnostic and Therapeutic Insights. Front Immunol (2020) 11:595835. doi: 10.3389/fimmu.2020.595835

77. Hong Q, Zhang L, Fu J, Verghese DA, Chauhan K, Nadkarni GN, et al. LRG1 Promotes Diabetic Kidney Disease Progression by Enhancing TGF-BetaInduced Angiogenesis. J Am Soc Nephrol (2019) 30:546-62. doi: 10.1681/ ASN.2018060599

78. Huang W, Ding X, Li B, Fan M, Zhou T, Sun H, et al. Serum Biomarkers Analyzed by LC-MS/MS as Predictors for Short Outcome of Non-Small Cell Lung Cancer Patients Treated With Chemoradiotherapy. Neoplasma (2013) 60:11-8. doi: 10.4149/neo_2013_002 
79. Shen WQ, Rong GQ, Wu Y, Pu YW, Ye ZY, Cao C, et al. Preliminary Proteomic Analysis of Radiation Response Markers in Rectal Cancer Patients. Eur Rev Med Pharmacol Sci (2019) 23:8841-51. doi: 10.26355/ eurrev_201910_19279

80. Zhang H, Zhou Y, Cheng Q, Dai Z, Wang Z, Liu F, et al. PDIA3 Correlates With Clinical Malignant Features and Immune Signature in Human Gliomas. Aging (Albany NY) (2020) 12:15392-413. doi: 10.18632/aging.103601

81. Farhad M, Rolig AS, Redmond WL. The Role of Galectin-3 in Modulating Tumor Growth and Immunosuppression Within the Tumor Microenvironment. Oncoimmunology (2018) 7:e1434467. doi: 10.1080/ 2162402X.2018.1434467

82. Chauvin A, Wang CS, Geha S, Garde-Granger P, Mathieu AA, Lacasse V, et al. The Response to Neoadjuvant Chemoradiotherapy With 5-Fluorouracil in Locally Advanced Rectal Cancer Patients: A Predictive Proteomic Signature. Clin Proteomics (2018) 15:16. doi: 10.1186/s12014-018-9192-2

83. Sumita K, Lo YH, Takeuchi K, Senda M, Kofuji S, Ikeda Y, et al. The Lipid Kinase PI5P4Kbeta Is an Intracellular GTP Sensor for Metabolism and Tumorigenesis. Mol Cell (2016) 61:187-98. doi: 10.1016/j.molcel.2015.12.011

84. Calon A, Lonardo E, Berenguer-Llergo A, Espinet E, Hernando-Momblona X, Iglesias M, et al. Stromal Gene Expression Defines Poor-Prognosis Subtypes in Colorectal Cancer. Nat Genet (2015) 47:320-9. doi: 10.1038/ng.3225

85. Skvortsova I, Skvortsov S, Stasyk T, Raju U, Popper BA, Schiestl B, et al. Intracellular Signaling Pathways Regulating Radioresistance of Human Prostate Carcinoma Cells. Proteomics (2008) 8:4521-33. doi: 10.1002/ pmic. 200800113

86. Khan I, Gril B, Steeg PS. Metastasis Suppressors NME1 and NME2 Promote Dynamin 2 Oligomerization and Regulate Tumor Cell Endocytosis, Motility, and Metastasis. Cancer Res (2019) 79:4689-702. doi: 10.1158/00085472.CAN-19-0492

87. Chang L, Ni J, Beretov J, Wasinger VC, Hao J, Bucci J, et al. Identification of Protein Biomarkers and Signaling Pathways Associated With Prostate Cancer Radioresistance Using Label-Free LC-MS/MS Proteomic Approach. Sci Rep (2017) 7:41834. doi: 10.1038/srep41834

88. Chang YC, Chiou J, Yang YF, Su CY, Lin YF, Yang CN, et al. Therapeutic Targeting of Aldolase A Interactions Inhibits Lung Cancer Metastasis and Prolongs Survival. Cancer Res (2019) 79:4754-66. doi: 10.1158/00085472.CAN-18-4080

89. Hao J, Graham P, Chang L, Ni J, Wasinger V, Beretov J, et al. Proteomic Identification of the Lactate Dehydrogenase A in a Radioresistant Prostate
Cancer Xenograft Mouse Model for Improving Radiotherapy. Oncotarget (2016) 7:74269-85. doi: 10.18632/oncotarget.12368

90. Cassim S, Vucetic M, Zdralevic M, Pouyssegur J. Warburg and Beyond: The Power of Mitochondrial Metabolism to Collaborate or Replace Fermentative Glycolysis in Cancer. Cancers (Basel) (2020) 12:1119. doi: 10.3390/ cancers 12051119

91. Newman EA, Abdessalam S, Aldrink JH, Austin M, Heaton TE, Bruny J, et al. Update on Neuroblastoma. J Pediatr Surg (2019) 54:383-9. doi: 10.1016/ j.jpedsurg.2018.09.004

92. Abou-Antoun TJ, Nazarian J, Ghanem A, Vukmanovic S, Sandler AD. Molecular and Functional Analysis of Anchorage Independent, TreatmentEvasive Neuroblastoma Tumorspheres With Enhanced Malignant Properties: A Possible Explanation for Radio-Therapy Resistance. PloS One (2018) 13: e0189711. doi: 10.1371/journal.pone.0189711

93. Hu X, Moscinski LC. Cdc2: A Monopotent or Pluripotent CDK? Cell Prolif (2011) 44:205-11. doi: 10.1111/j.1365-2184.2011.00753.x

94. Ray Chaudhuri A, Nussenzweig A. The Multifaceted Roles of PARP1 in DNA Repair and Chromatin Remodelling. Nat Rev Mol Cell Biol (2017) 18:610-21. doi: $10.1038 / \mathrm{nrm} .2017 .53$

95. Dapic I, Baljeu-Neuman L, Uwugiaren N, Kers J, Goodlett DR, Corthals GL. Proteome Analysis of Tissues by Mass Spectrometry. Mass Spectrom Rev (2019) 38:403-41. doi: 10.1002/mas.21598

Conflict of Interest: The authors declare that the research was conducted in the absence of any commercial or financial relationships that could be construed as a potential conflict of interest.

Publisher's Note: All claims expressed in this article are solely those of the authors and do not necessarily represent those of their affiliated organizations, or those of the publisher, the editors and the reviewers. Any product that may be evaluated in this article, or claim that may be made by its manufacturer, is not guaranteed or endorsed by the publisher.

Copyright (C) 2022 Luo and Ge. This is an open-access article distributed under the terms of the Creative Commons Attribution License (CC BY). The use, distribution or reproduction in other forums is permitted, provided the original author(s) and the copyright owner(s) are credited and that the original publication in this journal is cited, in accordance with accepted academic practice. No use, distribution or reproduction is permitted which does not comply with these terms. 